\title{
IDENTIFICAÇÃO E ANÁLISE QUALITATIVA DOS MÉTODOS DE AVALIAÇÃO DE IMPACTO AMBIENTAL CORRELACIONADOS COM A RESOLUÇÃO CONAMA N. 001:1986
}

\author{
Josiane de Almeida Aguiar - josiambiental@gmail.com \\ Universidade de Estado do Pará. \\ Lucimar Costa Pereira - lu.costa0708@gmail.com \\ Universidade de Estado do Pará. \\ Cinthia Carina Pereira Silva - ckpsilva@gmail.com \\ Universidade de Estado do Pará.
}

\section{RESUMO}

$\mathrm{Na}$ atualidade, as pressões sobre os ecossistemas têm aumentado sistematicamente em uma escala global, comprometendo a capacidade de suporte dos serviços ecossistêmicos. Desta forma, a realização da avaliação de impactos ambientais (AIA), torna-se imprescindível, pois a mesma, é definida como uma metodologia de avaliação, no qual compreende-se por um conjunto de métodos direcionados para identificação de fatores ambientais e de impactos, orientados para os mais variados propósitos e situações, sendo relativo sua utilização para cada situação.

O presente objetiva identificar e analisar qualitativamente os principais métodos de avaliação de impacto ambiental que podem ser usados para cumprimento dos critérios básicos e diretrizes para uso e implementação da AIA, estabelecidos pela Resolução CONAMA n. 001:1986. A prática de trabalho adotada nesse estudo foi qualitativa e indireta, com um levantamento de dados documentais no recorte temporário de 2008 a 2016. A partir da verificação em tal legislação, foram identificados os métodos: AD HOC, 


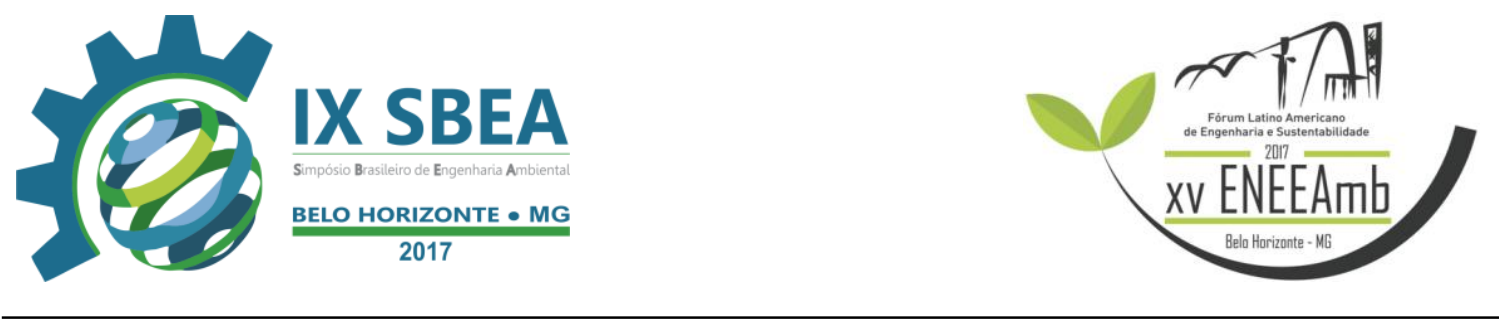

check-list, matrizes de interação, método redes de interação, superposição de cartas e modelo de simulação. Entre os métodos que apresentam maior número de conformidade com os incisos da Resolução CONAMA n. 001:1986 estar a matriz de interação, o que indica que a mesma é adequada para a execução da AIA, quando é levado em consideração, seus fundamentos, voltado para a sustentabilidade.

Palavras-chave: Avaliação, Método, Impacto Ambiental.

\section{INTRODUÇÃO}

Nas últimas décadas do século XXI, as pressões sobre os ecossistemas têm aumentado sistematicamente em uma escala global. Estes vêm se exaurindo e ameaçando a capacidade de suporte dos serviços providos pelos mesmos às atividades humanas. Desta forma, a Organização Mundial da Saúde (OMS) tem realizado alertas quanto a utilização não sustentável dos ecossistemas. Este uso ineficiente eleva o potencial de mudanças ecológicas para um quadro grave e irreversível (SILVEIRA; ARAUJO NETO, 2014).

O comprometimento de serviços ecossistêmicos é uma problemática mundial que precisa ser avaliada de forma concreta e eficiente. Neste contexto cabe a realização da avaliação de impactos ambientais (AIA), que possuem como um dos instrumentos de implementação o Estudo de Impactos Ambientais (EIA). Este deverá efetuar um estudo detalhado dos efeitos que um projeto preconizado irá causar no meio, verificando-se, dessa forma, a viabilidade ambiental de empreendimentos (FIGUEIREDO, et al., 2016: JOHNSON, 2012:).

A AIA, definida como uma metodologia de avaliação é compreendida por um conjunto de métodos direcionados para identificação de fatores ambientais e de impactos. Existem diversos tipos de ferramentas utilizáveis para auxiliar uma equipe na tarefa de identificar os impactos ambientais. Tais instrumentos foram desenvolvidos para facilitar o trabalho dos analistas, mas não se tratam de "pacotes" acabados (SÁNCHEZ, 2008).

Existem diversos métodos de avaliação de impacto ambiental, direcionados para os mais variados propósitos e situações, entretanto, é necessário o esclarecimento de que não existe um que se aplique a todo e qualquer estudo, pois nenhum é completo para a execução de uma avaliação de impactos. Todos apresentam potencialidades e limitações 
e a escolha dos mesmos para aplicação em cada caso depende de inúmeros fatores, tais como recursos técnicos e financeiros disponíveis, tempo para realização do estudo disponibilidade de dados e requisitos legais, características intrínsecas do tipo de empreendimento e produtos finais pretendidos (CASTRO, 2015).

A realização da AIA depende dos critérios estabelecidos em conteúdo Legislativo no contexto ambiental brasileiro. O mesmo inclui as Leis 6.803:1980 e 6938:1981, decretos, tais como n. 88.351:1983 e n. 99.274:1990. Abrange ainda algumas Resoluções do Conselho Nacional de Meio Ambiente (CONAMA), como exemplos a n. 237:1997 e 001:1986. Esta última apresenta conteúdo especifico referente a realização da AIA.

Desta forma, o presente trabalho objetiva identificar e analisar qualitativamente os principais métodos de avaliação de impacto ambiental que podem ser usados para cumprimento dos critérios básicos e diretrizes para uso e implementação da AIA, estabelecidos pela Resolução CONAMA n. 001:1986.

\section{MATEDOLOGIA}

A prática de trabalho adotada nesse estudo foi qualitativa e indireta a partir de revisões bibliográficas em trabalhos acadêmicos e Legislações referentes a Avaliação de Impactos Ambientais e os métodos da mesma. O levantamento de dados documentais está compreendido no recorte temporário de 2008 a 2016.

\section{RESULTADOS E DISCUSSÃO}

A partir da verificação da Legislação referente a AIA, especificamente a Resolução CONAMA n. 001:1986, foram identificados os métodos de avaliação de Impactos ambientais que podem ser utilizados para cumprimento dos critérios e diretrizes estabelecidos nos artigos 5, 6 e 7 da referida resolução, quadro 1.

Quadro 1: Relação entre os métodos de AIA e a resolução CONAMA 001:1986

\begin{tabular}{|c|c|c|c|c|c|c|c|}
\hline \multicolumn{2}{|c|}{ RESOLUÇÃO CONAMA 01:1986 } & \multicolumn{6}{|c|}{ MÉTODOS } \\
\hline Artigos & Incisos & A & $\mathrm{B}$ & $\mathrm{C}$ & $\mathrm{D}$ & $\mathrm{E}$ & $\mathrm{F}$ \\
\hline \multirow{4}{*}{ Art.5 } & I & & $\mathrm{X}$ & & & $\mathrm{X}$ & $\mathrm{X}$ \\
\hline & II & & & $\mathrm{X}$ & & & \\
\hline & III & & $\mathrm{X}$ & & & $\mathrm{X}$ & \\
\hline & IV & & & & & & \\
\hline & & & & & & & \\
\hline
\end{tabular}




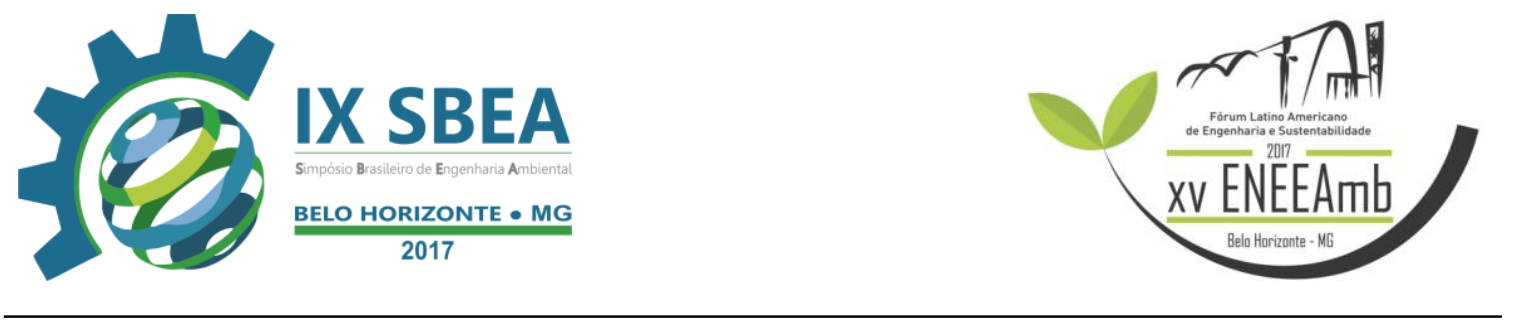

\begin{tabular}{|c|c|c|c|c|c|c|c|}
\multirow{4}{*}{ Art. 6 } & $\mathrm{I}$ & & $\mathrm{X}$ & $\mathrm{X}$ & & & \\
\cline { 2 - 8 } & $\mathrm{II}$ & & & $\mathrm{X}$ & $\mathrm{X}$ & & \\
\cline { 2 - 8 } & $\mathrm{III}$ & & & $\mathrm{X}$ & $\mathrm{X}$ & & \\
\cline { 2 - 8 } & $\mathrm{IV}$ & & & $\mathrm{X}$ & & & \\
\hline & & & & & & & \\
\hline Art.7 & & $\mathrm{X}$ & $\mathrm{X}$ & $\mathrm{X}$ & $\mathrm{X}$ & & \\
\hline
\end{tabular}

Legenda
A: Método AD HOC
B: Método Check-List: (listagem)
C: Método Matrizes de Interação
D: Método Redes de Interação
E: Método Superposição de Cartas: E
F: Método Modelo de Simulação: F

\subsection{MÉTODOS IDENTIFICADOS}

\subsubsection{AD HOC}

Este método é adequado para situações com pouca disponibilidade de dados, de modo que a principal fonte de informação é o conhecimento empírico de pessoas experientes no assunto, as quais trabalham de forma multidisciplinar. $\mathrm{O}$ mesmo desenvolve a avaliação de forma simples e objetiva e caracteriza-se por fazer a estimativa rápida dos possíveis impactos ambientais que podem ocorrer (COSTA et al., 2008).

Apenas o artigo 7 da Resolução CONAMA 001:1986 se encaixa nas características desse método, em razão da eficiência na identificação dos impactos ambientais, pois o mesmo estabelece que o estudo de impacto ambiental será realizado por equipe multidisciplinar (BRASIL, 1986). A aplicação deste método para cumprimento da legislação deve ser analisada de forma cuidadosa, haja vista que o mesmo não realiza um exame detalhado das intervenções e variáveis ambientais envolvidas, provocando um alto grau de subjetividade de seus resultados (CARVALHO\&LIMA, 2010).

\subsubsection{Check-List (listagem)}

Através da diagnose ambiental, esse método visa identificar e enumerar os impactos dos meios físico, biótico e socioeconômico. Os especialistas deverão relacionar os impactos decorrentes das fases de implantação e operação do empreendimento, 


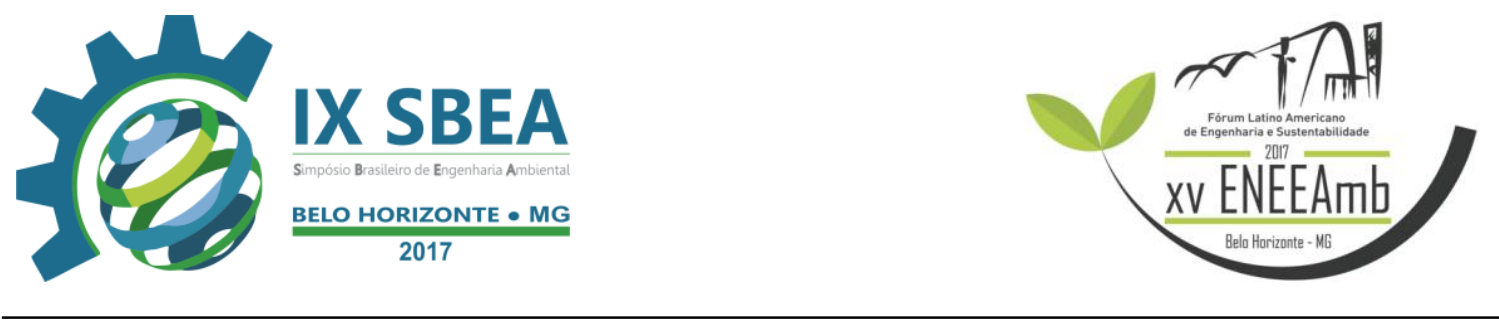

categorizando-os em positivos ou negativos, conforme o tipo da modificação antrópica que esteja sendo introduzida no sistema analisado (LOPES, 2012).

Esse método se encaixa em diversas exigências da Resolução CONAMA 01:1986 como exibido no quadro 1, entre elas, vale destacar o Art. 6, inciso I. Este estabelece as atividades técnicas mínimas, para o desenvolvimento de estudo de impacto ambiental, representado pelo diagnóstico ambiental da área de influência do projeto, completa descrição e análise dos recursos ambientais e suas interações, tal como existem, de modo a caracterizar a situação ambiental da área, antes da implantação do projeto, considerando os aspectos físico, biológico e sócio- econômico (BRASIL, 1986).

Desse modo, pode-se observar que de acordo com as características citadas anteriormente referentes ao método Check-List, o conteúdo do art. 6 da Resolução CONAMA abordada neste trabalho torna-se passível de execução. Entretanto, o conteúdo completo do Art.6 não pode ser cumprido somente pela execução do método de listagem, haja vista que assim como o AD HOC é passível de subjetividade.

\subsubsection{Matrizes de Interação}

Nesse método, as técnicas são usadas com o objetivo de identificar as interações entre os fatores ambientais e os componentes do projeto. É importante para verificar ações que causam maiores impactos ambientais. Existem vários tipos de matrizes, entre elas, a mais conhecida é a de Leopold. A referida Matriz é baseada em uma lista de 100 ações com potencial de possíveis provedores de impacto ambiental e 88 características ambientais (FINUCCI, 2010).

Nas Matrizes de Interação, faz-se necessário inicialmente, assinalar todas as possíveis interações entre as ações e os fatores, para que posteriormente se estabeleça a magnitude e a importância de cada impacto em uma escala que varia de 1 a 10. A partir disto, é possível identificar e avaliar se o impacto em questão é positivo ou negativo (OLIVEIRA\&MOURA, 2009: VALDETARO et al., 2015).

Os dados obtidos no quadro 1 mostram que o método de Matrizes se encaixa em um maior número de exigências estabelecidas pela resolução CONAMA 001:1986, o que indica que o mesmo é eficiente no processo de avaliação de impacto ambiental, pois a partir da identificação e classificação dos impactos (negativo ou positivo), é possível se 


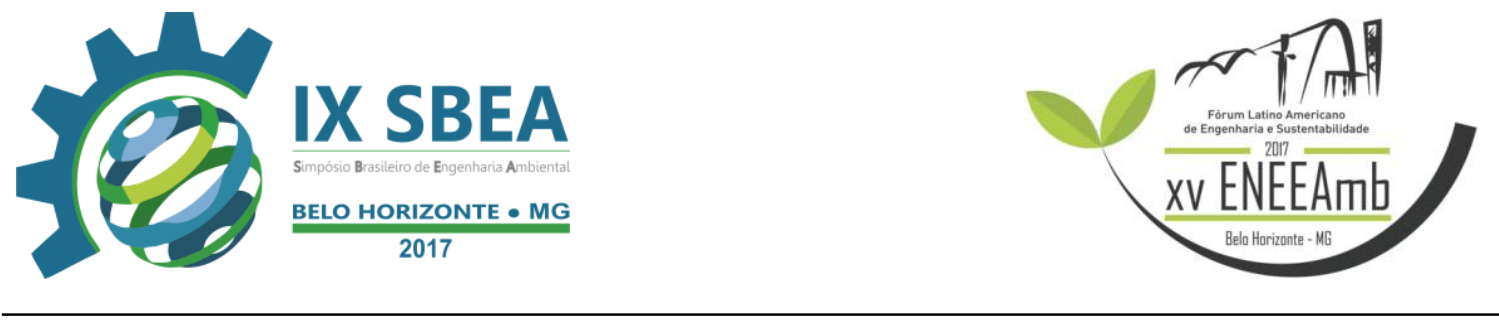

estabelecer as medidas mitigatórias para estes, bem como facilitar a elaboração de programas de acompanhamento e monitoramento.

Para o método em questão, na correlação com a legislação citada anteriormente, ressalta-se o art. 6. O referido artigo com todos os seus incisos, estabelecem diretrizes para realização de um diagnóstico ambiental, análise dos impactos ambientais do projeto e de suas alternativas, a partir da identificação, previsão da magnitude e interpretação da importância dos prováveis impactos relevantes, definição das medidas mitigadoras dos impactos negativos e elaboração do programa de acompanhamento e monitoramento, (BRASIL, 1986).

\subsubsection{Método Redes de Interação}

Esse método tem por objetivo fazer uma relação de precedência entre ações praticadas pelo empreendimento e os consequentes impactos de primeira e demais ordens. Além disso, possuem a vantagem de possibilitar a introdução de parâmetros probabilísticos, mostrando tendências (LOPES, 2012). Tal método consiste em uma maneira de organizar as informações em uma tabela disposta na forma de uma rede, onde as atividades do projeto se apresentam num eixo e as características ambientais em outro (SILVA\&MORAES, 2012).

O quadro 1 mostra que as Redes de Interação podem ser usadas para cumprimento das exigências estabelecidas no Art. 6, nos incisos II e III bem como no Art.7. Com relação ao primeiro artigo mencionado e o seus respectivo incisos, a exigência consiste em fazer análise dos impactos ambientais do projeto por meio da identificação, previsão da magnitude bem como da importância dos prováveis impactos relevantes a longo prazo (BRASIL, 1986). Atendido esse critério, o método, auxilia na elaboração de medidas mitigatórias, o que encaixa-o no inciso III do mesmo artigo.

Para fazer a identificação dos impactos ambientais decorrentes das ações, a partir da interação de redes, é necessário que haja a participação de uma equipe multidisciplinar, haja vista que há possibilidade de cruzamento de disciplinas, com a execução de análises em uma mesma cadeia de impactos, dos efeitos sobre a economia, fauna, água, dentre outros recursos (CREMONEZ, 2014). Tal característica permite que o método seja passível de aplicação no cumprimento da diretriz estabelecida no Art. 7 da Resolução 
CONAMA 001:1986, o qual estabelece que o EIA será realizado por equipe multidisciplinar habilitada (BRASIL, 1986).

\subsubsection{Superposição de Cartas}

O método superposição de Cartas foi adaptado para ser aplicado na AIA e desenvolvido afim de atender a demanda do planejamento territorial. O procedimento mais comum e utilizado consiste na justaposição de transparências em um conjunto de mapas que estabelece o impacto individual de uma região para obter um panorama do impacto a nível global. Em cada mapa, as características culturais, sociais e físicas são indicadas e refletem um impacto sobre o meio. A produção dos mapas é realizada a partir de fotos aéreas, observação do terreno e com o auxílio de programas do Sistema de Informação Geográfica (SIG) e profissionais da área (MARTINHA, 2013).

Esse método pode ser utilizado para cumprimento do que é estabelecido nos incisos I e II do art. 5 da Resolução CONAMA 001: 1986, conforme mostra o quadro 1. Esta evidência pode ser verificada quando se analisa o conteúdo dos incisos, que estabelecem que o estudo de impacto ambiental deve contemplar todas as alternativas tecnológicas e de localização do projeto e o propõem que o estudo faça a definição dos limites da área geográfica a ser direta ou indiretamente afetada pelos impactos (BRASIL, 1986). Nesse contexto o método obedece às duas exigências, já que contempla as alternativas tecnológicas e favorece a identificação dos limites geográficos.

\subsubsection{Modelo de Simulação}

Este método consiste em modelos de simulações computadorizadas com o uso de inteligência artificial ou modelos matemáticos, destinados a reproduzir tanto quanto possível o comportamento de parâmetros ambientais ou as inter-relações entre as causas e os efeitos de determinadas ações (OLIVEIRA; MOURA, 2009).

Os modelos de simulação mais usados e reproduzidos consistem naqueles feitos para realização de estimativas de impactos provocados pelas emissões gasosas e de lançamento de efluentes no meio ambiente. Nestes, há a incorporação de hipóteses e pressuposição em prol dos processos e relações entre os fatores bióticos, físicos e culturais em relação ás alterações causadas palas ações que devem ser avaliadas (ROCHA et al., 2012). 


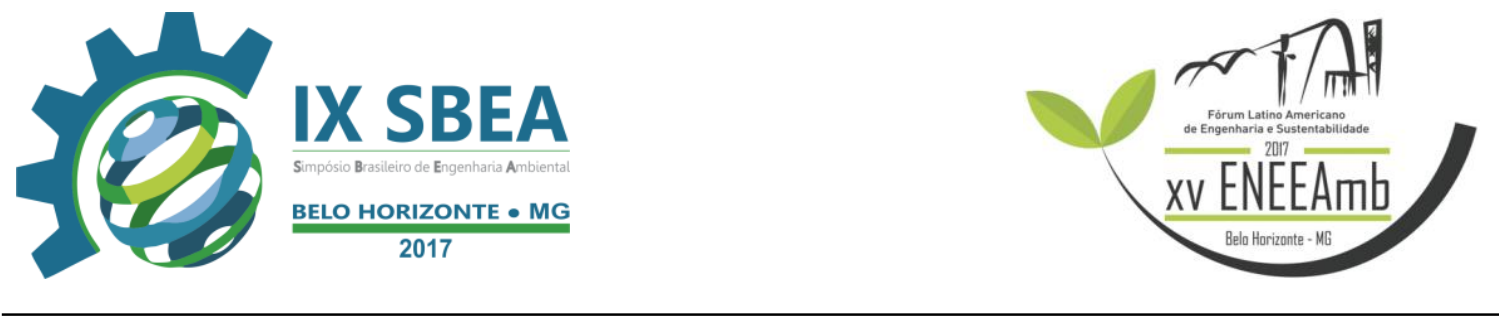

As características de aplicação desse método permitem que o mesmo seja usado para cumprimento do conteúdo estabelecido no inciso I, Art. 5 da resolução abordada no quadro 1. Tal inciso estabelece que o estudo de impacto ambiental deve contemplar todas as alternativas tecnológicas e de localização de projeto, ou seja, está correlacionado com o conteúdo característico do modelo de simulação.

\section{CONCLUSÃO}

Entre os métodos que apresentam maior número de conformidade com os incisos da Resolução CONAMA 001:1986 estar a matriz de interação, o que indica que a mesma é adequada para a execução da AIA, quando é levado em consideração, seus fundamentos, voltado para a sustentabilidade. Um dos fatores que tornam esse método eficiente é a sua complexidade em que, nesse processo, são integrados os âmbitos econômico, social e ambiental.

Quanto aos demais métodos, a principal falha evidenciada foi a subjetividade. Tal fator pode ser um desempasse para atender a realização do AIA, pois, abre margem para a predominância de grupos de interesses. Essa problemática, indica que há a necessidade de novos métodos de AIA, que venham ser efetivamente sustentáveis e que atendam os fundamentos da PNRS, e assim se enquadrem em maior números de incisos das legislações vigentes.

\section{REFERÊNCIAS}

BRASIL. Decreto n. 99.274 de 6 de junho de 1990. Regulamenta a Lei no 6.902, de 27 de abril de 1981, e a Lei ${ }^{\circ}$ 6.938, de 31 de agosto de 1981, que dispõem, respectivamente sobre a criação de Estações Ecológicas e Áreas de Proteção Ambiental e sobre a Política Nacional do Meio Ambiente, e dá outras providências. Diário Oficial da União, Brasília, DF, 6 jun. 1990.

BRASIL. Decreto n. Decreto $\mathrm{n}^{\circ} 88.351$, de $1^{\circ}$ de Junho de 1983. Regulamenta a Lei $\mathrm{n}^{\circ}$ 6.938, de 31 de agosto de 1981, e a Lei n ${ }^{\circ}$ 6.902, de 27 de abril de 1981, que dispõem, respectivamente, sobre a Política Nacional do Meio Ambiente e sobre a criação de Estações Ecológicas e Áreas de Proteção Ambiental, e dá outras providências. Diário Oficial da União, Brasília, 01 jun. 1983. 


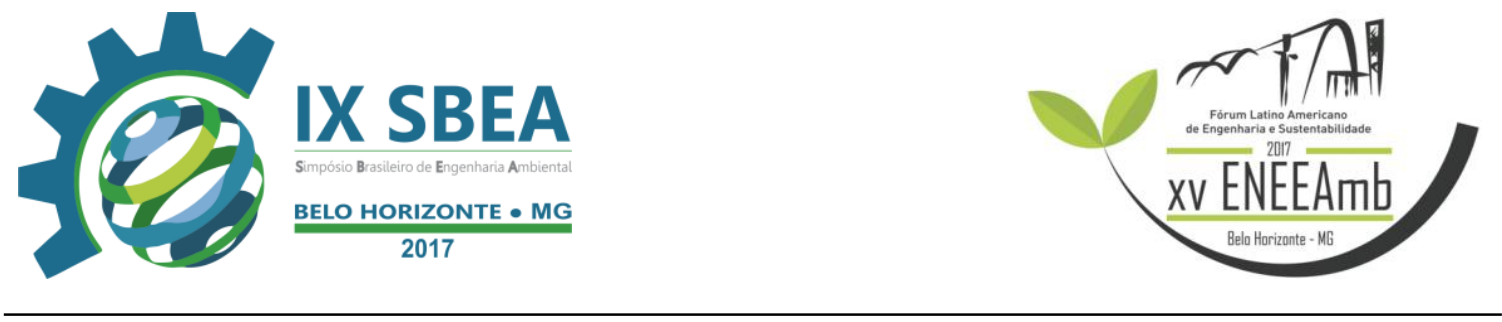

BRASIL. Lei n. 6.803, 02 de julho de 1980. Dispõe sobre as diretrizes básicas para o zoneamento industrial nas áreas críticas de poluição, e dá outras providências. Brasília DF: 1980 .

BRASIL. Lei $n^{\circ} .6 .938$ de 31 de agosto de 1981. Dispõe sobre a Política Nacional do Meio Ambiente, seus fins e mecanismos de formulação e aplicação, e dá outras providências. Diário Oficial da República Federativa do Brasil, Brasília, DF, 1981.

BRASIL. Resolução 001 de 23 janeiro de 1986. Dispõe sobre critérios básicos e diretrizes gerais para o Relatório de Impacto Ambiental - RIMA. Disponível em: www.soleis.adv.br.Acesso em: 18/04/2017.

CARVALHO, D.L. de; LIMA, A. V. de. Metodologias para Avaliação de Impactos Ambientais de Aproveitamentos Hidrelétricos. In: ENCONTRO NACIONAL DE GEÓGRAFOS, 16., 2010, Porto Alegre. Anais.... Porto Alegre: Agb, 2010. p. 1 - 11.

CASTRO, M. B., et al. Metodologias para avaliação de impactos ambientais. In: CONGRESSO NACIONAL DE MEIO AMBIENTE DE POÇOS DE CALDAS, 12., 2015, Poços de Caldas. Anais...Poços de Caldas: GSC Eventos Especiais, 2015. p. 1 - 7.

COSTA, M. V.; CHAVES, P. S. V.; OLIVEIRA, F. C. Uso das Técnicas de Avaliação de Impacto Ambiental em Estudos Realizados no Ceará. In: CONGRESSO BRASILEIRO DE CIÊNCIAS DA COMUNICAÇÃO, 28., 2008, Rio de Janeiro. Anais.... Rio de Janeiro: Intercom, 2008. p. 1 - 15.

CREMONEZ, F. E. et al. Avaliação de impacto ambiental: metodologias aplicadas no Brasil. Revista Monografias Ambientais, Santa Maria, v. 13, n. 5, p.3821-3830, 16 nov. 2014. Universidad Federal de Santa Maria.

FIGUEIREDO, H.P. et al. Environmental impacts of public policies in Campo Grande, MS: the case of Lajeado stream environmental protection area. Interações, Campo Grande, v. 17, n. 4, p. 713-728, out./dez. 2016.

JOHNSON, A.B. Avaliação e Gestão de Impactos Ambientais de infraestrutura hídrica em projetos de irrigação. Monografia. Engenharia Civil (mestrado), Universidade Federal do Rio Grande do Sul, 2012.

LOPES, A. J. Identificação dos aspectos e impactos ambientais dos processos produtivos de uma empresa de construções mecânicas. 2012. 95 f. Dissertação (Mestrado) - Curso de Engenharia Ambiental, Universidade Federal de Santa Catarina, Florianópolis, 2012.

MARTINHA, D. D. Metodologias utilizadas na avaliação de impacto ambiental. Revista Varia Scientia Agrárias, Paraná, v. 04, n. 01, p.146-156, abr. 2013. 
OLIVEIRA, F.C.; MOURA, H.J.T. de. Uso das metodologias de avaliação de impacto ambiental em estudos realizados no Ceará. Pretexto, Belo Horizonte, v.10, n.4, p.79-98, Out/dez. 2009.

ROCHA, M. V. L. et al. Análise comparativa entre medições in situ e estimativas numéricas na Praia da Cornélia, Costa da Caparica, Portugal. Revista da Gestão Costeira Integrada, Lisboa, v. 12, n. 2, p.147-157, maio 2012.

SÁNCHEZ, L.E. Avaliação de Impacto Ambiental: conceitos e métodos. São Paulo: Oficina de textos, 2008.

SILVEIRA, M.; ARAUJO NETO, M. D. Licenciamento ambiental de grandes empreendimentos: conexão possível entre saúde e meio ambiente. Ciência \& Saúde Coletiva, Brasília, v. 19, n. 9, p.3829-3838, set. 2014.

VALDETARO, E. B. et al. Conjugação dos métodos da matriz de interação e do checklist na avaliação quali-quantitativa de impactos ambientais de um programa de fomento florestal. Revista Árvore, Viçosa, v. 39, n. 4, p.611-622, ago. 2015. 